\title{
Decreased telomere length in the small airway epithelium suggests accelerated aging in the lungs of persons living with human immunodeficiency virus (HIV)
}

\author{
Stella Xu1, Emily A. Vucic ${ }^{2}$, Tawimas Shaipanich³, Stephen Lam², Wan Lam², Julio S. Montaner ${ }^{4}$, Don D. Sin ${ }^{1,3}$, \\ S. F. Paul Man ${ }^{1,3}$ and Janice M. Leung ${ }^{1,3^{*}}$
}

\begin{abstract}
Human immunodeficiency virus (HIV) infection is associated with an increased risk of chronic obstructive pulmonary disease (COPD) independent of cigarette smoke exposure. Previous studies have demonstrated that decreased peripheral leukocyte telomere length is associated with HIV, suggesting an accelerated aging phenomenon. We demonstrate that this process of telomere shortening also occurs in the lungs, with significant decreases in telomere length observed in small airway epithelial cells collected during bronchoscopy. Molecular evidence of accelerated aging in the small airway epithelium of persons living with HIV may be one clue into the predisposition for chronic lung disease observed in this population.
\end{abstract}

Keywords: HIV, Telomere length, Epithelial cell

\section{To the editor}

Persons living with human immunodeficiency virus (PLWH) face a growing burden of age-related conditions including chronic obstructive pulmonary disease (COPD) [1]. One proposed etiology for this phenomenon is the concept of "inflamm-aging" [2] whereby PLWH, even those on chronic antiretroviral therapy, continue to have low-grade inflammation driving cellular turnover. An important biomarker of this particular form of accelerated biologic aging is telomere length, a measure of replicative senescence. Telomeres, made up of repeated TTAGGG sequences, are the ends of chromosomes that provide structural integrity during the process of cell division. With each division cycle, the fidelity of replicating these ends is imperfect with the loss of approximately 25 to 200 base pairs (bp) with each division [3]. Ultimately, a critical telomere length is reached whereby cell proliferation

\footnotetext{
* Correspondence: janice.leung@hli.ubc.ca

${ }^{1}$ Centre for Heart Lung Innovation, University of British Columbia, Vancouver, Canada

${ }^{3}$ Division of Respiratory Medicine, University of British Columbia, Vancouver, Canada

Full list of author information is available at the end of the article
}

ceases and apoptosis ensues. We have previously demonstrated that peripheral leukocyte telomere lengths in PLWH are significantly shorter than in HIV-negative control subjects, with the severity of lung function as measured by forced expiratory volume in 1 second (FEV1) correlating with decreased telomere length [4]. In this study, we further these findings by determining whether advanced replicative senescence can also be observed within the lungs of PLWH.

PLWH were drawn from the patient population at St. Paul's Hospital in Vancouver, Canada, a tertiary care setting with an active bronchoscopy program and HIV outpatient clinic. Eligible PLWH were patients with documented HIV-1 infection who were undergoing bronchoscopies for clinical purposes (i.e. for lung masses or nodules or to rule out infection) with additional research specimen collection added on to the procedure. All subjects were $\geq 19$ years old and provided written informed consent under the University of British Columbia (UBC) Providence Health Care ethics protocol H14-03267. HIV-negative control subjects were recruited from participants of a lung cancer screening study at the British Columbia Cancer Agency in Vancouver, Canada. With the 
exception of three PLWH who were lost to follow up, subjects underwent spirometry according to American Thoracic Society/European Respiratory Society guidelines using the Crapo reference equations [5]. 24 PLWH and 41 HIV-negative participants were enrolled.

Small airway epithelial (SAE) cells were obtained in regions of the lung that were free from lung nodules, masses, or infiltrates, as determined by chest computed tomography scans. Cytologic brushings were taken via flexible bronchoscopy, with brushings occurring in the order of the 5th-6th generation airways where resistance to the brush was met. Airway epithelial cell telomere length was measured by quantitative polymerase chain reaction methods originally outlined by O'Callahan and Fenech [6]. Standard curves were generated from known quantities of synthesized oligomers of telomere (TEL) DNA [(TTAG GG) ${ }_{14}$ ] and single copy gene (36B4) DNA [CAGCAAGTG GGAAGGTGTAATCCGTCTCCACAGACAAGGCCAGG ACTCGTTTGTACCCGTTGATGATAGAATGGG]. Sample telomere DNA length was then measured based on the ratio of telomere DNA length to 36B4 (RPLPO) DNA length as obtained from their respective curves.

Differences in demographic characteristics between PLWH and the HIV-negative cohort were assessed using t-tests or Mann-Whitney $U$ tests for continuous variables (depending on the normality of distribution) and Fisher's exact tests for categorical variables. The relationships between telomere length and continuous variables were determined using Pearson correlation tests. T-tests were used to evaluate telomere length differences between PLWH and HIV-negative controls in a univariate model. Given differences in demographics between the two cohorts with respect to age, sex distribution, and smoking pack-years, a linear regression model adjusting for these factors was performed to determine whether the differences between PLWH and HIV-negative telomere lengths persisted. Because the PLWH cohort was predominantly male, a secondary analysis was performed using only male participants.

Demographic characteristics of the cohort are listed in Table 1. PLWH were significantly younger with a greater proportion of males. Lung function as measured by FEV1/ forced vital capacity (FVC) and FEV1 \%Predicted was similar between the two groups as were the proportion of current, former, and never smokers. HIV-negative patients had significantly greater smoking pack-years. $71 \%$ of PLWH had an undetectable viral load with a mean CD4 count of 462 cells $/ \mathrm{mm}^{3}$.

Despite the fact that PLWH were on average 4 years younger than HIV-negative patients, their telomere lengths were significantly shorter $(108.13 \pm 45.51$ kilobase pair (kbp)/genome for PLWH, $171.53 \pm 52.20 \mathrm{kbp} /$ genome for HIV-negative patients, $p<0.001)$. This relationship remained significant $(p<0.001)$ after adjustment for sex,
Table 1 Characteristics of the study cohort

\begin{tabular}{llll}
\hline Characteristic & PLWH $(n=24)$ & HIV- $(n=41)$ & $p$-value \\
\hline Age & $58.13 \pm 9.14$ & $62.59 \pm 6.70$ & 0.044 \\
Male sex (\%) & $20(83 \%)$ & $23(56 \%)$ & 0.031 \\
FEV1 (\% Predicted) & $76.79 \pm 15.84$ & $76.24 \pm 24.02$ & 0.579 \\
FEV1/FVC (\%) & $68.57 \pm 12.70$ & $66.39 \pm 15.43$ & 0.697 \\
Smoking status & & & \\
$\quad$ Current & $12(50 \%)$ & $19(46 \%)$ & 0.184 \\
$\quad$ Former & $10(42 \%)$ & $22(54 \%)$ & \\
$\quad$ Never & $2(8 \%)$ & 0 & \\
Smoking Pack-Years & $29.54 \pm 31.09$ & $48.98 \pm 13.53$ & $<0.001$ \\
Undetectable Plasma & $17(71 \%)$ & - & - \\
HIV Viral Load & & & - \\
CD4 Count (cells/mm $\left.{ }^{3}\right)$ & $462 \pm 306$ & - & $<0.001$ \\
$\begin{array}{l}\text { Telomere Length } \\
\text { (kbp/genome) }\end{array}$ & $108.13 \pm 45.51$ & $171.53 \pm 52.20$ & \\
\hline
\end{tabular}

smoking pack-years, and age. Given the differences in sex distribution between the two groups and the previously reported differences in telomere lengths between males and females, a second analysis was performed with just male participants (mean age 59.96 years for male PLWH and 60.73 years for HIV-negative males, $p=0.724$ ). Male PLWH still had significantly shorter telomere lengths compared to HIV-negative males $(104.45 \pm 44.67 \mathrm{kbp} /$ genome vs. $178.51 \pm 52.84 \mathrm{kbp} /$ genome, $p<0.001)$. After adjustment for age and smoking pack-years, the relationship between HIV serostatus and shorter telomere length in only males remained significant $(p<0.001)$.

The relationships between telomere length and other variables of interest are shown in Tables 2 and 3. Consistent with a previous study in which smoking appears to attenuate the relationship between age and telomere length in SAE cells, telomere length in our predominantly smoking cohort was not significantly correlated with age [7]. Telomere length was also not significantly correlated with FEV1/FVC, FEV1 \%Predicted, COPD status (as defined by a FEV1/FVC <70\%), smoking pack-years, or sex. Within PLWH, telomere length was not different between those with detectable and undetectable viral loads. There was also no correlation between telomere length and CD4 count.

Table 2 Telomere length associations with continuous variables

\begin{tabular}{lll}
\hline Variable & $R$ & $p$-value \\
\hline Age & 0.089 & 0.479 \\
Smoking Pack-Years & 0.118 & 0.353 \\
FEV1/FVC & -0.040 & 0.760 \\
FEV1\% Predicted & -0.068 & 0.604 \\
CD4 cell count (PLWH only) & -0.015 & 0.946 \\
\hline
\end{tabular}


Table 3 Telomere length associations with dichotomous variables

\begin{tabular}{lcc}
\hline Variable & Telomere length mean \pm SD (kbp/genome) & $p$-value \\
\hline Sex & $144.07 \pm 61.33$ & \\
Male & $156.06 \pm 52.27$ & 0.414 \\
Female & $104.77 \pm 70.74$ & \\
Viral Load (PLWH Only) & 0.561 \\
Detectable $\quad 116.29 \pm 48.10$ & \\
Undetectable & & \\
COPD (all patients) & 0.510 \\
Yes & $158.311 \pm 56.09$ & \\
No & $148.45 \pm 59.01$ & \\
COPD (PLWH only) & $104.94 \pm 43.08$ & \\
Yes & $119.62 \pm 52.17$ & \\
No & $174.56 \pm 49.59$ & \\
COPD (HIV-negative only) & \\
Yes & $167.66 \pm 56.58$ & \\
No & &
\end{tabular}

In this study, we provide the first molecular evidence for accelerated aging within the SAE of PLWH, many of whom had abnormal lung function. The difference in telomere length was nearly double what was found between healthy male smokers and non-smokers in a previous study looking at SAE telomere lengths [7]. Importantly, the majority of PLWH in this study had sufficient immune recovery and undetectable plasma HIV viral loads, and our findings were significant even after accounting for cigarette smoke exposure. Two possible mechanisms might explain our observations: first that HIV accelerates epithelial cell turnover in the small airway or second that HIV impacts the telomere lengths of basal stem/progenitor cells that give rise to the SAE. Either of these possibilities may be the result of the virus itself. The effect of HIV on the SAE has recently been investigated in two studies. One suggests that the presence of X4-tropic virus increases epithelial cell permeability and the expression of pro-inflammatory cytokines [8], while another has found that HIV interacts with the basal epithelial layer to create a tissue-destructive phenotype driven by matrix metalloproteinases [9]. Whether HIV can replicate within the epithelial cell is a matter of contention $[8,10]$, regardless, one effect on the SAE layer may be the promotion of a particularly injurious and inflammatory environment by which senescence mechanisms are triggered. As shortened telomere lengths are a measure of replicative senescence, our work supports this hypothesis. Future work into functionally characterizing the senescent nature of these cells and the impact of their presence on surrounding lung parenchymal, stromal, and immune cells is ongoing and of great interest.
The question of whether or not replicative senescence in the SAE contributes to the development of future COPD within PLWH requires larger prospective and longitudinal studies. Unlike a previous study from our group in which peripheral leukocyte telomere length was associated with lung function in PLWH, we did not find decrements in lung function to be significantly associated with SAE telomere shortening. We suspect that our small sample size may have left us underpowered to detect such a signal or that the distribution of lung function in our cohort skewed towards more severe disease. Repeat measurements in a larger cohort with a wide distribution of lung function should be performed. Investigation of telomere length in asymptomatic PLWH without apparent lung disease and in HIV cohorts with a greater proportion of females will also be critical in extending these findings to larger populations. Moreover, other measurements of aging such as methylation changes, senescence-associated beta-galactosidase, and DNA damage markers may provide further evidence of a global aging process in HIV. In summary, we describe here an important aging phenotype within a critical cell layer of the small airway that may distinguish PLWH from their uninfected counterparts.

\section{Abbreviations}

COPD: Chronic obstructive pulmonary dsiease; FEV1: Forced expiratory volume in 1 second; FVC: Forced vital capacity; HIV: Human immunodeficiency virus; PLWH: Persons living with HIV; SAE: Small airway epithelium; TL: Telomere length

\section{Funding}

This study was funded by the Canadian Institutes for Health Research and the British Columbia Lung Association. JML is supported by the Michael Smith Foundation for Health Research, the Canadian Institutes for Health Research, and the St. Paul's Foundation.

Availability of data and materials

The dataset used and analysed during the current study is available from the corresponding author on reasonable request.

\section{Authors' contributions}

Study Concept: JML, DDS, SFPM. Study Design: JML, DDS, SFPM. Acquisition of Data: EAV, SL, WL, SX, TS, JM, JML, DDS, SFPM. Analysis and Interpretation of Data: JML, SX, DDS, SFPM. Manuscript Writing: JML. Manuscript Editing: EAV, SL, $J M L, J M, D D S, S F P M$. All authors read and approved the final manuscript.

\section{Ethics approval and consent to participate}

All subjects provided written informed consent under the University of British Columbia (UBC) Providence Health Care ethics protocol H14-03267. HIV- control subjects were recruited from participants of a lung cancer screening study at the British Columbia Cancer Agency in Vancouver, Canada.

Competing interests

The authors declare that they have no competing interests.

\section{Publisher's Note}

Springer Nature remains neutral with regard to jurisdictional claims in published maps and institutional affiliations. 


\section{Author details}

'Centre for Heart Lung Innovation, University of British Columbia, Vancouver, Canada. ${ }^{2}$ British Columbia Cancer Agency, Vancouver, Canada. ${ }^{3}$ Division of Respiratory Medicine, University of British Columbia, Vancouver, Canada. ${ }^{4} \mathrm{BC}$ Centre for Excellence in HIV/AIDS, University of British Columbia, Vancouver, Canada.

Received: 28 March 2018 Accepted: 31 May 2018

\section{Published online: 13 June 2018}

\section{References}

1. Crothers K, Huang L, Goulet $\mathrm{L}$, et al. HIV infection and risk for incident pulmonary diseases in the combination antiretroviral therapy era. Am J Respir Crit Care Med. 2011;183(3):388-95.

2. Franceschi C, Bonafe M, Valensin S, et al. Inflamm-aging. An evolutionary perspective on immunosenescence. Ann N Y Acad Sci. 2000;908:244-54.

3. Prasad KN, Wu M, Bondy SC. Telomere shortening during aging: attenuation by antioxidants and anti-inflammatory agents. Mech Ageing Dev. 2017:164:61-6.

4. Liu JC, Leung JM, Ngan DA, et al. Absolute leukocyte telomere length in HIV-infected and uninfected individuals: evidence of accelerated cell senescence in HIV-associated chronic obstructive pulmonary disease. PLoS One. 2015;10(4):e0124426.

5. Crapo RO, Morris AH, Gardner RM. Reference spirometric values using techniques and equipment that meet ATS recommendations. Am Rev Respir Dis. 1981;123(6):659-64

6. O'Callaghan NJ, Fenech M. A quantitative PCR method for measuring absolute telomere length. Biol Proced Online. 2011;13:3.

7. Walters MS, De BP, Salit J, et al. Smoking accelerates aging of the small airway epithelium. Respir Res. 2014;15:94.

8. Brune KA, Ferreira F, Mandke P, et al. HIV impairs lung epithelial integrity and enters the epithelium to promote chronic lung inflammation. PLoS One. 2016;11(3):e0149679.

9. Chung NPY, Ou X, Khan KMF, Salit J, Kaner RJ, Crystal RG. HIV reprograms human airway basal stem/progenitor cells to acquire a tissue-destructive phenotype. Cell Rep. 2017;19(6):1091-100.

10. Chinnapaiyan S, Parira T, Dutta R, et al. HIV infects bronchial epithelium and suppresses components of the Mucociliary clearance apparatus. PLoS One. 2017;12(1):e0169161.

\section{Ready to submit your research? Choose BMC and benefit from:}

- fast, convenient online submission

- thorough peer review by experienced researchers in your field

- rapid publication on acceptance

- support for research data, including large and complex data types

- gold Open Access which fosters wider collaboration and increased citations

- maximum visibility for your research: over $100 \mathrm{M}$ website views per year

At BMC, research is always in progress.

Learn more biomedcentral.com/submissions 